\title{
Path planning for simple wheeled robots: Sub-Riemannian and elastic curves on SE(2)
}

\author{
Craig Maclean* James D. Biggs \\ Advanced Space Concepts Laboratory, Department of Mechanical \& Aerospace Engineering, \\ University of Strathclyde, Glasgow, G4 OLT, UK
}

\begin{abstract}
This paper presents a motion planning method for a simple wheeled robot in two cases: (i) where translational and rotational speeds are arbitrary and (ii) where the robot is constrained to move forwards at unit speed. The motions are generated by formulating a constrained optimal control problem on the Special Euclidean group $S E(2)$. An application of Pontryagin's maximum principle for arbitrary speeds yields an optimal Hamiltonian which is completely integrable in terms of Jacobi elliptic functions. In the unit speed case, the rotational velocity is described in terms of elliptic integrals and the expression for the position reduced to quadratures. Reachable sets are defined in the arbitrary speed case and a numerical plot of the time-limited reachable sets presented for the unit speed case. The resulting analytical functions for the position and orientation of the robot can be parametrically optimised to match prescribed target states within the reachable sets. The method is shown to be easily adapted to obstacle avoidance for static obstacles in a known environment.
\end{abstract}

\footnotetext{
${ }^{*}$ Corresponding author. E-mail: craig.maclean@ $@$ strath.ac.uk
}

\section{Introduction}

The task of computing a suitable trajectory from a given initial condition to a desired final point is fundamental in robotics. However for some robotic systems, such as wheeled robots, motion planning is challenging due to their inherent nonholonomic constraints. Subsequently, many methods use linearisation or introduce local co-ordinates in order to tackle the motion planning problem (MPP). Nevertheless, motion planning is essentially a global problem and ideally requires a global approach.

In the context of optimisation the optimal path between the start and end points is often selected via the use of a numerical optimisation procedure. Despite the numerous optimisation tools available, the use of optimal control theory to tackle the MPP has had little impact on practical applications, presumably because the delicate numerical treatment of optimal control problems is often less suited to practical implementation than other methods.

However, since the development of geometric control theory ${ }^{1}$, new approaches have arisen which exploit the underlying analytic structure of the system. When the configuration space can be represented by a Lie group, 
as in the case of the wheeled robot on the Special Euclidean group $S E(2)^{2}$, motion planning algorithms can be designed based on this global representation ${ }^{3-5}$. For nonholonomic systems defined on Lie groups the motion planning problem methodologies are naturally based on Lie-algebraic techniques. The control functions are designed such that motions are generated in the direction of the iterated Lie bracket i.e. in the direction which is not directly controlled. By application of the maximum principle of optimal control ${ }^{6}$, and by specifying an appropriate quadratic cost function, the optimal Hamiltonian for nonholonomic systems such as a wheeled robot can be obtained. If the Hamiltonian is integrable then the optimal control problem can be reduced to quadratures and often solved analytically. Moreover, for wheeled robots whose configuration space is $\operatorname{SE}(2)$, the Hamiltonian is always integrable ${ }^{1}$.

The problem of motion planning for simple wheeled robots has been widely studied. In early work on the subject Dubins ${ }^{7}$ derived a method for generating trajectories for a car like robot, termed Dubins' car $^{8}$, which is constrained to move forwards at unit speed. In this method the paths are constructed from straight line segments and arcs of constant curvature, and are referred to as Dubins' curves or paths ${ }^{8}$. Dubins' curves are shortest length curves in Euclidean space, whose curvature is uniformly bounded for all points along the curve, which connect two arbitrary points ${ }^{9}$. It was shown that Dubins' car is capable of arriving at any state (assuming no obstacles) using a combination of no more than three motion primitives - left turn, right turn or straight ahead. Reeds and Shepp ${ }^{10}$ extended the work of Dubins by defining the shortest paths for a car capable additionally of reversing at unit speed. Dubins' curves have been used extensively in motion planning for a range of systems, including unmanned air vehicles ${ }^{11}$ and underwater vehicles ${ }^{12}$. Scheuer and Fraichard ${ }^{13}$ developed a motion planning method to overcome an inherent limitation of Dubins' curves - that the wheeled robots are required to stop to reorientate at each section of the path. This method, derived from Dubins' curves, generates continuous curvature curves which do not require the robot to stop and reorientate, and was extended to include obstacle avoidance $^{14,15}$.

Murray and Sastry ${ }^{16}$ extended the work of Brockett ${ }^{17}$ on steering drift free nonholonomic systems, and showed that for certain classes of system the optimal controls take the form of sinusoids. A particular benefit of this method is the ability to satisfy orientation constraints by exploiting the periodicity of the controls. Other methods for motion planning of wheeled robots include the use of polynomial spiral trajectories ${ }^{18}$, in which the path is described by a continuous control function.

Murray, Canny and Latombe ${ }^{19-21}$ pay particular attention to the nonholonomic motion planning problem for simple robots, while Brockett ${ }^{22}$ showed that for a particular nonholonomic system the optimal controls were elliptic functions.

In this paper a simple analytical motion planning method is derived via the framework of geometric control theory that seeks to define a general class of curves for the wheeled robot in two cases (i) where translational and rotational speeds are arbitrary and (ii) where the robot is constrained to move forwards at unit speed. The optimal Hamiltonian is shown to be completely solvable in the arbitrary speed case, where the position and orientation can be described analytically using elliptic functions. These elliptic functions have special cases which include 
hyperbolic functions and the sinusoids commonly used in motion planning ${ }^{16}$. As a result, a wide range of possible curves are obtained. In the unit speed case the rotational velocity is described in terms of elliptic integrals and the expression for the position can be reduced to quadratures. A truncated approximation is then used to describe the position analytically. It is interesting to note that the motion planning formulation of a wheeled robot with arbitrary and unit speed is analogous to the definition of sub-Riemannian and elastic curves on $S E(2)$ respectively. Therefore, when tracking these motions the robotic vehicles will trace a sub-Riemannian or elastic curve.

Reachable sets are defined exactly in the arbitrary speed case, and time-limited reachable sets presented in the unit speed case. A method for parametrically optimising the analytical equations describing the motion to match prescribed boundary conditions and produce reference tracks is presented. The completely analytical expressions enable the references to be generated rapidly and at low computational cost. Finally it is shown that the set of feasible curves can be probed to create a simple obstacle avoidance algorithm in a static and known environment. This enables a range of single curves to be generated which match boundary conditions on the final position while avoiding forbidden areas.

The paper is organised as follows. In Section 2 we introduce the model of the wheeled robot and explain some preliminary concepts concerning the framework of geometric control theory. In Section 3 we explain how the optimal controls are derived from the optimal Hamiltonian. In Section 4 we solve explicitly for the completely analytical optimal controls in the arbitrary speed and unit speed cases. Reachable sets are defined in Section 5. In
Section 6 we describe the parametric optimisation procedure to find the optimal curve which satisfies the boundary conditions, and show how the procedure can be extended to provide simple obstacle avoidance for static and known obstacles. Finally we summarise the findings of the paper and present future work in Section 7.

\section{Problem Statement on SE(2)}

In this section the motion planning problem for a simple wheeled robot is framed in the context of an optimal control problem on a matrix Lie group. The general definitions of a Lie group, Lie algebra and the dual of the Lie algebra are introduced, and these concepts are related to the specific problem of motion planning for a wheeled robot.

\subsection{Model}

The wheeled robot model under consideration is shown in Figure 1. The distance between the front and

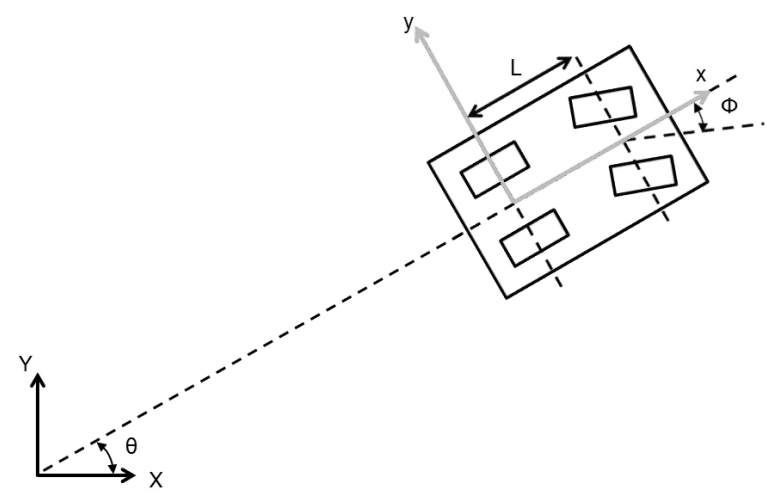

Figure 1: Wheeled robot model. Body axis is placed at centre of rear axle with $\mathrm{x}$-axis lying along centre line of robot.

rear axles is given by $L$. The angle between the body fixed $x-y$ frame and the inertial $X-Y$ frame is denoted by $\theta$. A sliding constraint is imposed by the assumption that in a small time interval $d t$ the wheeled robot moves in approximately the direction that the rear wheels are pointing ${ }^{8}$. This condition can be written as the Pfaffian 
constraint:

$$
-\dot{X} \sin \theta+\dot{Y} \cos \theta=0
$$

As in Choset $^{23}$ we choose to focus on the position and orientation of the wheeled robot. Therefore we eliminate the steering angle $\phi$ from the representation of the configuration and treat it as part of the control. The control system is then described by:

$$
\begin{aligned}
& \dot{X}=u_{1} \cos \theta \\
& \dot{Y}=u_{1} \sin \theta \\
& \dot{\theta}=u_{3} ;
\end{aligned}
$$

where $u_{1}$ and $u_{3}$ are the controls in translation and rotation respectively.

\subsection{Preliminary Concepts}

We begin with some general definitions of matrix Lie groups, the Lie algebra and it's dual from Bloch ${ }^{2}$ :

Definition 1 A matrix Lie group is a set of invertible $n \times n$ matrices that are closed under matrix multiplication and that are a submanifold of $R^{n \times n}$.

An example of a matrix Lie group is the Special Euclidean group, SE(2), whose elements represent the configuration described by an orthonormal frame attached to a Euclidean plane.

Definition 2 A Lie algebra $\mathfrak{g}$ is a set of $n \times n$ matrices that is a vector space with respect to the usual operations of matrix addition and multiplication by real numbers (scalars) and that is closed under the matrix Lie bracket operation $[\cdot, \cdot]$.

Definition 3 Let $G$ be a Lie group and $\mathfrak{g}$ its Lie algebra with $[\cdot, \cdot]$ the associated Lie bracket. The dual space $\mathfrak{g}^{*}$ is a Poisson manifold with Poisson bracket $\{\hat{p}(\cdot), \hat{p}(\cdot)\}=$ $-\hat{p}([\cdot, \cdot])$ where $(\cdot) \in \mathfrak{g}$.
We will now relate these concepts to the optimal control of a nonholonomic wheeled robot. The configuration space of the wheeled robot in Figure 1 can be described by a curve $g(t) \in S E(2)$ and expressed in matrix form as:

$$
g(t)=\left(\begin{array}{cc}
R(t) & \gamma \\
0 & 1
\end{array}\right)
$$

where $R(t) \in S O(2)$ (the Special Orthogonal group) represents the rotational component of the motion, and $\gamma \in \mathfrak{R}^{2}$ (the Euclidean plane) the translational component. The rotation matrix $R(t)$ is given by:

$$
R(t)=\left(\begin{array}{cc}
\cos \theta & -\sin \theta \\
\sin \theta & \cos \theta
\end{array}\right)
$$

and $\gamma=\left[\begin{array}{ll}X & Y\end{array}\right]^{T}$.

The tangent space at the identity is the Lie algebra denoted $\mathfrak{s e}(2)$. It follows that the kinematics of the simple wheeled robot can be expressed as a left invariant control system on $\operatorname{SE}(2)$ as:

$$
\frac{d g(t)}{d t}=g(t)\left(\sum_{i=1}^{s} u_{i}(t) A_{i}\right)
$$

where $u_{i}$ with $i=1, \ldots, s$ are controls and $A_{1}, \ldots, A_{3} \in$ $\mathfrak{s e}(2)$ are the basis of the Lie algebra $\mathfrak{s e}(2)$. The Lie algebra $\mathfrak{s e}(2)$ is a vector space together with the matrix commutator, the Lie bracket:

$$
[X, Y]=X Y-Y X
$$


where $X, Y \in \mathfrak{s e}(2)$. The basis are defined by:

$$
\begin{aligned}
& A_{1}=\left(\begin{array}{lll}
0 & 0 & 1 \\
0 & 0 & 0 \\
0 & 0 & 0
\end{array}\right) \\
& A_{2}=\left(\begin{array}{lll}
0 & 0 & 0 \\
0 & 0 & 1 \\
0 & 0 & 0
\end{array}\right) \\
& A_{3}=\left(\begin{array}{ccc}
0 & -1 & 0 \\
1 & 0 & 0 \\
0 & 0 & 0
\end{array}\right)
\end{aligned}
$$

satisfying the commutative table shown in Table 1.

Table 1: Commutative table for basis on $\mathfrak{s e}(2)$

\begin{tabular}{|c|c|c|c|}
\hline & $A_{1}$ & $A_{2}$ & $A_{3}$ \\
\hline$A_{1}$ & 0 & 0 & $-A_{2}$ \\
\hline$A_{2}$ & 0 & 0 & $A_{1}$ \\
\hline$A_{3}$ & $A_{2}$ & $-A_{1}$ & 0 \\
\hline
\end{tabular}

Note that the lateral direction, represented by the basis $A_{2}$, is not directly controlled $\left(u_{2}=0\right)$ due to the sliding constraint expressed in (1). However the Lie bracket enables motions to be generated in the $A_{2}$ direction despite not having a control directly associated with it.

By differentiating (3) and substituting into (5) we obtain the control system defined in (2). Note also that the driftless system in (5) can be augmented to include systems with drift by setting one of the controls $u_{i}$ to a constant a priori without loss of generality.

Subject to the kinematic nonholonomic constraint given by (5) and given that the system is controllable the problem is then to find a trajectory $g(t) \in S E(2)$ from an initial position and orientation $g(0) \in S E(2)$ to a final position and orientation $g(T) \in S E(2)$, where $T$ is some fixed final time, that minimises the functional:

$$
J=\frac{1}{2} \int_{0}^{T} \sum_{i=1}^{s} c_{i} u_{i}^{2} d t
$$

where $i=1, \ldots, s$ and $c_{i}$ are constant weights. In addition it enables the MPP to be formulated in the context of geometric optimal control and this enables us to ask questions of integrability and in some cases solve the system in closed form. Furthermore, obtaining a closed form solution essentially reduces the MPP to a problem of optimising the available parameters to match the prescribed boundary conditions.

\section{Methodology}

The methodology for the MPP comprises the following phases:

1. Lifting the optimal control problem on $S E(2)$ to a Hamiltonian setting via the maximum principle of optimal control.

2. Solving integrable cases of the Hamiltonian vector fields analytically in the most general form of the cost function (8).

3. Given the optimal velocities derive the corresponding motions in $S E(2)$ analytically, reducing the MPP to a parameter optimisation problem.

4. Defining the reachable sets of the optimal motions.

5. As the boundary conditions are not contained in the cost function, optimise the available parameters of the analytic solutions to match the prescribed boundary conditions.

The application of the coordinate free maximum principle to left-invariant optimal control problems is well 
known, see Jurdjevic ${ }^{1}$ and Sussman ${ }^{24}$. As the Hamiltonian is left-invariant the cotangent bundle $T^{*} S E(2)$ can be realised as the direct product $S E(2) \times \mathfrak{s e}(2)^{*}$ where $\mathfrak{s e}(2)^{*}$ is the dual of the Lie algebra $\mathfrak{s e}(2)$ of $S E(2)$. Therefore, the original Hamiltonian defined on $T^{*} S E(2)$ can be expressed as a reduced Hamiltonian on the dual of the Lie algebra $\mathfrak{s e}(2)^{*}$ as $T^{*} S E(2) / S E(2) \cong \mathfrak{s e}(2)^{*}$. Essentially this means that the translational and rotational symmetry of the wheeled robot problem enables the Hamiltonian to be defined independently of configuration co-ordinates on the dual of the Lie algebra. This means that the Hamiltonian is highly simplified, and makes the process of solving for the optimal controls simpler.

The appropriate Hamiltonian for the constraint (5) with respect to minimizing the cost function (8) is given by (see Jurdjevic ${ }^{1}$ for details):

$$
H(p, u, g)=\sum_{i=1}^{s} u_{i} p\left(g(t) A_{i}\right)-\rho_{0} \frac{1}{2} \sum_{i=1}^{s} c_{i} u_{i}^{2}
$$

where $s \leq 3, p \in T^{*} S E(2)$ and $\rho_{0}=1$ for regular extremals and $\rho_{0}=0$ for abnormal extremals. Jurdjevic ${ }^{1}$ showed that abnormal extremals are a subset of regular extremals, therefore in this paper we consider only the regular extremals, setting $\rho_{0}=1$. As stated the Hamiltonian (9) defined on $T^{*} S E(2)$ is expressed as a reduced Hamiltonian on the dual of the Lie algebra $\mathfrak{s e}(2)^{*}$. It follows that $p\left(g(t) A_{i}\right)=\hat{p}\left(A_{i}\right)$ for any $p=(g(t), \hat{p})$ and any $A_{i} \in \mathfrak{s e}(2)$. Defining the extremal (linear) functions explicitly as $\lambda_{i}=\hat{p}\left(A_{i}\right)$, where $\hat{p} \in \mathfrak{s e}^{*}(2)$ the Hamiltonian (9) can be expressed on $\mathfrak{s e}(2)^{*}$ as:

$$
H=\sum_{i=1}^{s} u_{i} \lambda_{i}-\frac{1}{2} \sum_{i=1}^{s} c_{i} u_{i}^{2}
$$

Through the maximum principle and the fact that the control Hamiltonian (10) is a concave function of the control functions $u_{i}$, it follows by calculating $\frac{\partial H}{\partial u_{i}}=0$ that the optimal kinematic control inputs are:

$$
u_{i}^{*}=\frac{1}{c_{i}} \lambda_{i}
$$

where $i=1, \ldots, s$ and $\lambda_{i}$ are the extremal curves. Substituting (11) back into (10) gives the appropriate leftinvariant quadratic Hamiltonian:

$$
H=\frac{1}{2}\left(\sum_{i=1}^{s} \frac{\lambda_{i}^{2}}{c_{i}}\right)
$$

For each quadratic Hamiltonian (12), the corresponding vector fields are calculated using the Poisson bracket $\{\hat{p}(\cdot), \hat{p}(\cdot)\}=-\hat{p}([\cdot, \cdot])$ where $(\cdot) \in \mathfrak{s e}(n)$. Then the Hamiltonian vector fields are given by:

$$
\frac{d(\cdot)}{d t}=\{\cdot, H\}
$$

where $(\cdot) \in \mathfrak{s e}(2)^{*}$. Finally, substituting (11) into (5) yields:

$$
\frac{d g(t)}{d t}=g(t) \nabla H
$$

where $\nabla H$ is the gradient of the Hamiltonian and $g(t) \in$ $S E(2)$ are the corresponding paths. The MPP is thus reduced to solving for $g(t) \in S E(2)$ such that the boundary conditions $g(0) \in S E(2)$ and $g(T) \in S E(2)$ in some final time $T$ are matched. Equations (13) and (14) are integrable with the three integrals of motion: (i) the Hamiltonian $H$; (ii) the Casimir function $M=\lambda_{1}^{2}+\lambda_{2}^{2}$ and ; (iii) the integral of motion $\varphi_{3}$ corresponding to a rightinvariant vector field. The corresponding Hamiltonian vector-fields (13) which describe the extremal curves are 3-dimensional with the Hamiltonian and Casimir providing two integrals of motion. Therefore each extremal can be decoupled and described by a 1 degree of freedom ordinary differential equation. In addition integrability is an intrinsic property of the system as it implies that all motions will be regular. 


\section{Extremal Curves}

In this section we derive the optimal Hamiltonians in the arbitrary speed and unit speed cases through application of Pontryagin's maximum principle, before solving explicitly for the optimal controls.

\subsection{Arbitrary Translational and Rotational Speed}

We assume that the wheeled robot can move backward or forwards at a velocity $v$ which can be controlled. This sliding constraint (1) can be expressed as:

$$
\frac{d \gamma}{d t}=R(t)\left[\begin{array}{l}
v \\
0
\end{array}\right]
$$

where $\gamma=\left[\begin{array}{ll}X & Y\end{array}\right]^{T}$. Furthermore, the robot can rotate at an angular velocity $\omega=\dot{\theta}$ which can also be controlled. Differentiating equation (3) and taking into the account the constraint (15) it is easily shown that the nonholonomic kinematic constraint can be expressed as a left-invariant differential equation:

$$
g(t)^{-1} \frac{d g(t)}{d t}=\left(\begin{array}{ccc}
0 & -\omega & v \\
\omega & 0 & 0 \\
0 & 0 & 0
\end{array}\right)
$$

This can be expressed in the form:

$$
g(t)^{-1} \frac{d g(t)}{d t}=v A_{1}+\omega A_{3}
$$

where the Lie algebra is given by (7) and the cost function (8) is given by:

$$
J=\frac{1}{2} \int_{0}^{1} v^{2}+c \omega^{2} d t
$$

where $c$ is a constant weight. The time $t$ is scaled such that in real time $\tau$ with final fixed time $T$ is $t=\tau / T$. In relation to the general form (8) $u_{1}=v, c_{1}=1, u_{3}=$ $\omega, c_{3}=c$. This cost function minimises steering effort and forward velocity. Together, the kinematic constraints
(16) and the cost (18) are analogous to a sub-Riemannian curve on $\operatorname{SE}(2)^{25}$. That is, while we constrain the motion planning problem to a fixed time $T$, a curve would be constrained by a fixed length. Therefore the Hamiltonian function corresponding to the constraint (16) that minimises the cost function (18) is:

$$
H=v \lambda_{1}+\omega \lambda_{3}-\frac{1}{2}\left(v^{2}+c \omega^{2}\right)
$$

Then Pontryagin's maximum principle says that if:

$$
\frac{\partial H}{\partial v}=0, \frac{\partial H}{\partial \omega}=0, \frac{\partial^{2} H}{\partial v^{2}}<0, \frac{\partial^{2} H}{\partial \omega^{2}}<0,
$$

then the functions $v$ and $\omega$ are optimal. These conditions are satisfied if:

$$
v=\lambda_{1}, \quad \omega=\frac{\lambda_{3}}{c}
$$

Substituting these values into (19) yields the optimal Hamiltonian $H^{*}$ :

$$
H^{*}=\frac{1}{2}\left(\lambda_{1}^{2}+\frac{\lambda_{3}^{2}}{c}\right)
$$

The corresponding Hamiltonian vector fields which implicitly define the extremal solutions are given by the Poisson bracket $\frac{d \lambda_{i}}{d t}=\left\{\lambda_{i}, H\right\}$ where $i=1, \ldots, 3$. This yields the differential equations:

$$
\begin{aligned}
& \dot{\lambda}_{1}=\frac{\lambda_{2} \lambda_{3}}{c} \\
& \dot{\lambda}_{2}=-\frac{\lambda_{1} \lambda_{3}}{c} \\
& \dot{\lambda}_{3}=-\lambda_{1} \lambda_{2}
\end{aligned}
$$

In addition observe that the Casimir function:

$$
M=\lambda_{1}^{2}+\lambda_{2}^{2}
$$

is constant along the Hamiltonian flow i.e. $\left\{M, H^{*}\right\}=0$. The integrability of the system can be confirmed geometrically by plotting the intersection of the integrals of the system, the Hamiltonian (22) and the Casimir function (24), as shown in Figure 2. 


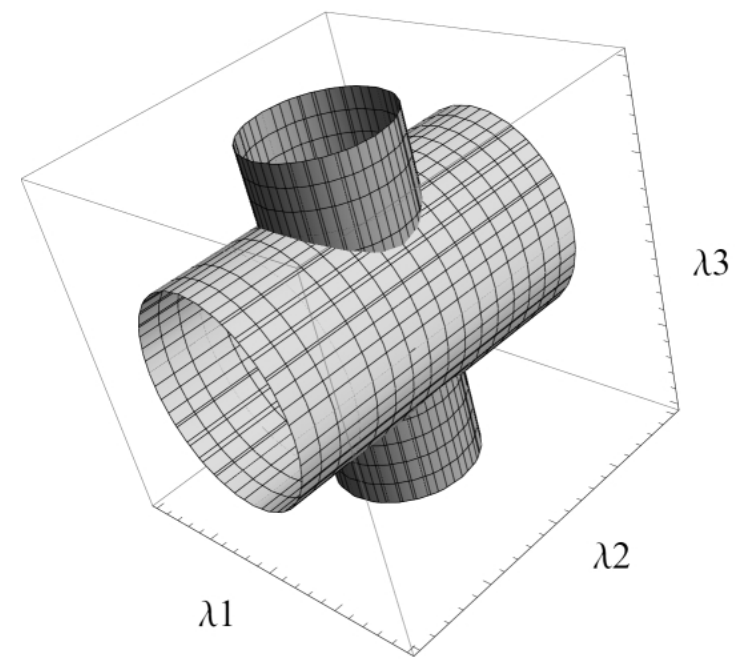

Figure 2: Plot of intersection between the Hamiltonian function (light) and Casimir (dark) in arbitrary speed case. In this case $m=M / 2 H^{*}<1$

It is known that the intersection of two quadratic surfaces define elliptic curves which in turn are parameterised by elliptic functions, see Husemoller ${ }^{26}$. Therefore the extremal curves can be solved via elliptic functions as shown in the following Lemma:

Lemma 1 The optimal velocity $v$ in the surge direction and angular velocity $\omega$ that minimise the cost function (18) subject to the kinematic constraint (16) are Jacobi elliptic functions $\operatorname{sn}(\cdot, \cdot), d n(\cdot, \cdot)$ of the form:

$$
\begin{aligned}
& v=\sqrt{M} s n\left(\frac{\sqrt{2} H^{*}}{\sqrt{c H^{*}}} t, \frac{M}{2 H^{*}}\right) \\
& \omega=\sqrt{\frac{2 H^{*}}{c}} d n\left(\frac{\sqrt{2} H^{*}}{\sqrt{c H^{*}}} t, \frac{M}{2 H^{*}}\right)
\end{aligned}
$$

where $H^{*}$ and $M$ are constants defined by (22) and (24) respectively and $c$ is the constant weight in the cost function (18) with the corresponding path:

$$
\begin{aligned}
& X=-\frac{\sqrt{2} \sqrt{c H^{*}}}{\sqrt{M}} d n \Phi+\sqrt{\frac{2 H^{*} c}{M}} \\
& Y=\frac{2 H^{*} t}{\sqrt{M}}-\sqrt{2 H^{*} c} E\left(\operatorname{am} \Phi, \frac{M}{2 H^{*}}\right)
\end{aligned}
$$

where $E(\cdot, \cdot)$ is the elliptic integral of the second kind and am $(\cdot)$ is the Jacobi amplitude and where the rotation of the body along the path is:

$$
R(t)=\left(\begin{array}{cc}
c n \Phi & -s n \Phi \\
s n \Phi & c n \Phi
\end{array}\right)
$$

with $\Phi=\left(\frac{\sqrt{2} H^{*}}{\sqrt{c H^{*}}} t, \frac{M}{2 H^{*}}\right)$, and:

$$
\theta=\operatorname{am}(\Phi)
$$

Proof.

The conserved quantity (24) can be parameterised by the Jacobi elliptic functions:

$$
\lambda_{1}=r s n(\alpha t, m), \quad \lambda_{2}=r c n(\alpha t, m)
$$

Substituting (29) into (24) yields $r=\sqrt{M}$. Then (22) can be parameterised by defining:

$$
\lambda_{3}=\operatorname{adn}(\alpha t, m)
$$

Substituting (29) and (30) into (22) we find that $a=$ $\sqrt{2 H^{*} c}$. From this we can derive that $m=M / 2 H^{*}$ and so:

$$
\begin{aligned}
& \lambda_{1}=r s n\left(\alpha t, \frac{M}{2 H^{*}}\right) \\
& \lambda_{2}=\operatorname{rcn}\left(\alpha t, \frac{M}{2 H^{*}}\right) \\
& \lambda_{3}=\sqrt{2 H^{*}} \operatorname{dn}\left(\alpha t, \frac{M}{2 H^{*}}\right)
\end{aligned}
$$

Finally to obtain $\alpha$ substitute (31) into (23), giving $\alpha=$ $\sqrt{2} H^{*} / \sqrt{c H^{*}}$ and enabling the complete expressions for the extremal functions to be written as:

$$
\begin{aligned}
& \lambda_{1}=r s n\left(\frac{\sqrt{2} H^{*}}{\sqrt{c H^{*}}} t, \frac{M}{2 H^{*}}\right) \\
& \lambda_{2}=\operatorname{rcn}\left(\frac{\sqrt{2} H^{*}}{\sqrt{c H^{*}}} t, \frac{M}{2 H^{*}}\right) \\
& \lambda_{3}=\sqrt{2 H^{*} c} d n\left(\frac{\sqrt{2} H^{*}}{\sqrt{c H^{*}}} t, \frac{M}{2 H^{*}}\right)
\end{aligned}
$$

The relationship between the optimal velocities and the extremals (21) then enables us to derive (25). As $\omega=\dot{\theta}$ 
it follows from (25) that:

$$
\theta=\operatorname{am}(\Phi)+C_{1}
$$

where $C_{1}$ is a constant of integration. For simplicity we set $C_{1}=0$ such that the rotation matrix $R(t)$ emanates from the origin. This yields (27). Substituting (27) and (25) into equation (15) we find that:

$$
\frac{d \gamma}{d t}=\left[\begin{array}{c}
\sqrt{M} \operatorname{si} \Phi c n \Phi \\
\sqrt{M} s n^{2} \Phi
\end{array}\right]
$$

These can be integrated analytically to give the expressions for the evolution of the robot's path (26).

Remark 1 It is interesting to note the change in behaviour of the elliptic functions as the parameter $m=$ $M / 2 H^{*}$ changes. For $0<m<1$ the optimal controls are described by (25), and the Casimir and Hamiltonian intersect as in Figure 2.

As $m=M / 2 H^{*} \rightarrow 0$ in (25), the velocity in translation tends to a sinusoid and the velocity in rotation tends to a constant, viz:

$$
\begin{aligned}
& v=\sqrt{M} \sin \left(\frac{\sqrt{2} H^{*}}{\sqrt{c H^{*}}} t\right) \\
& \omega=\sqrt{\frac{2 H^{*}}{c}}
\end{aligned}
$$

Note also that the elliptic functions in the expression (34) tend to sine and cosine. As sine and cosine are special cases of the elliptic functions described above, this suggests that it may be possible to derive a more general form of the control using sinusoids described by Murray and Sastry ${ }^{16}$.

As $m=M / 2 H^{*} \rightarrow 1$, the equations (25) tend to hyperbolic functions:

$$
\begin{aligned}
& v=\sqrt{M} \tanh \left(\frac{\sqrt{2} H^{*}}{\sqrt{c H^{*}}} t\right) \\
& \omega=\sqrt{\frac{2 H^{*}}{c}} \operatorname{sech}\left(\frac{\sqrt{2} H^{*}}{\sqrt{c H^{*}}} t\right)
\end{aligned}
$$

This case is shown in Figure 3a).

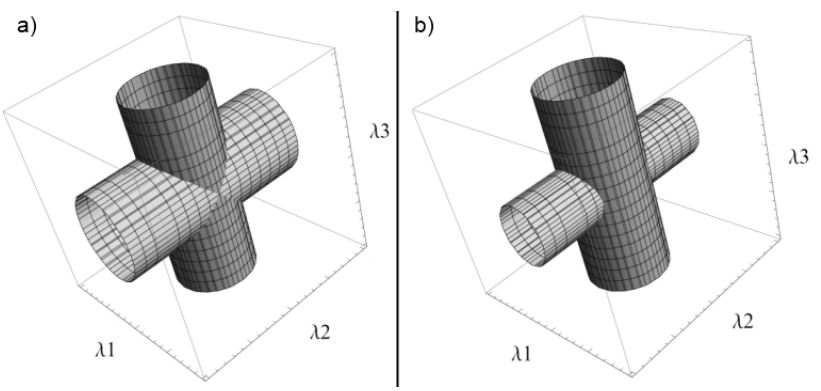

Figure 3: Plot of intersection between the Hamiltonian function (light) and Casimir (dark) in arbitrary speed case defining the extremal curves for a) $m=M / 2 H^{*}=1$ and b) $m=M / 2 H^{*}>1$

Finally when $m=M / 2 H^{*}>1$, the equations (25) are transformed using the Jacobi transformation ${ }^{27}$ which gives:

$$
\begin{aligned}
& v=\sqrt{2 H^{*}} \operatorname{sn}\left(\sqrt{\frac{M}{c}} t, \frac{2 H^{*}}{M}\right) \\
& \omega=\sqrt{\frac{2 H^{*}}{c}} c n\left(\sqrt{\frac{M}{c}} t, \frac{2 H^{*}}{M}\right)
\end{aligned}
$$

This case is shown in Figure 3b). The behaviour of the elliptic functions can be further explained by analogy with the simple pendulum ${ }^{28}$. Plotting the curves in Figures 2 and 3 in 2D we obtain Figure 4.

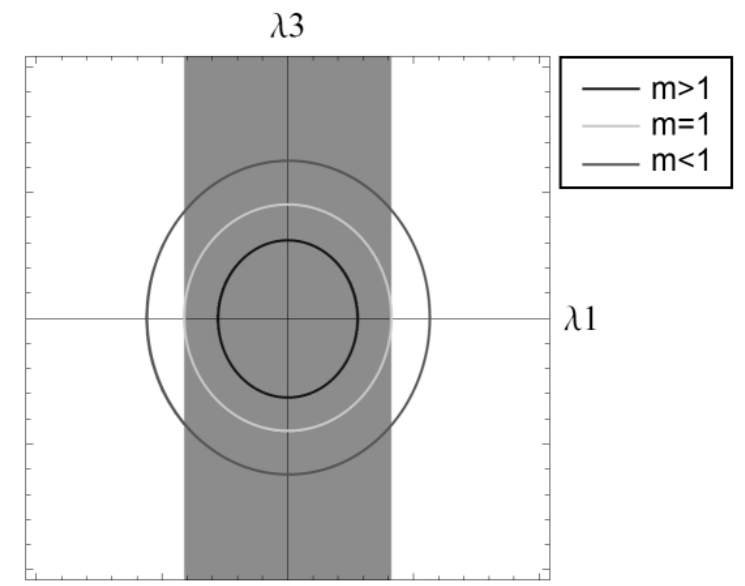

Figure 4: 2D plot of intersection between the Hamiltonian function and Casimir (solid shaded area) in arbitrary speed case for different values of $m$

The case where $m>1$ can be thought of as corresponding to oscillatory solutions in the phase plane of the pendulum where it is swinging back and forth, while $m=1$ de- 
fines a heteroclinic connection and $m<1$ the case where the pendulum has high energy.

\subsection{Unit Speed}

We now assume that the wheeled robot is constrained to move forward at fixed unit speed $(v=1)$. This corresponds to the case where the system has drift. The velocity constraint can be expressed as:

$$
\frac{d \gamma}{d t}=R(t)\left[\begin{array}{l}
1 \\
0
\end{array}\right]
$$

Furthermore, the robot can rotate at an angular velocity $\omega=\dot{\theta}$ which is controllable. Differentiating equation (3) and taking into the account the constraint (38) the nonholonomic kinematic constraint can be expressed as the left-invariant differential equation:

$$
g(t)^{-1} \frac{d g(t)}{d t}=\left(\begin{array}{ccc}
0 & -\omega & 1 \\
\omega & 0 & 0 \\
0 & 0 & 0
\end{array}\right)
$$

This can be written in the form:

$$
g(t)^{-1} \frac{d g(t)}{d t}=A_{1}+\omega A_{3}
$$

where the Lie algebra is given by (7) and the cost function (8) is expressed as:

$$
J=\frac{1}{2} \int_{0}^{1} \omega^{2} d t
$$

The time $t$ is scaled such that in real time $\tau$ with final fixed time $T$ is $t=\tau / T$. In relation to the general form (8) $u_{1}=1, c_{1}=1, u_{3}=\omega, c_{3}=1$. Together, the kinematic constraint (39) and the cost function (41) are analogous to the definition of an elastic curve on $\operatorname{SE}(2)$, where $\omega$ is analogous to curvature. Jurdjevic ${ }^{29}$ reduced the extremals of this problem to quadratures. However, this paper extends this by solving explicitly the optimal steering control in terms of an elliptic function.
The Hamiltonian function corresponding to the constraint (39) that minimises the cost function (41) is:

$$
H=\lambda_{1}+\omega \lambda_{3}-\frac{1}{2}\left(\omega^{2}\right)
$$

Then, Pontryagin's maximum principle says that if:

$$
\frac{\partial H}{\partial \omega}=0, \frac{\partial^{2} H}{\partial \omega^{2}}<0,
$$

then the function $u$ is optimal. These conditions are satisfied if:

$$
\omega=\lambda_{3}
$$

Substituting these values into (42) yields the optimal Hamiltonian $H^{*}$ :

$$
H^{*}=\frac{1}{2}\left(\lambda_{3}^{2}\right)+\lambda_{1}
$$

The corresponding Hamiltonian vector fields which implicitly define the extremal solutions are given by the Poisson bracket $\frac{d \lambda_{i}}{d t}=\left\{\lambda_{i}, H^{*}\right\}$. This yields the differential equations:

$$
\begin{aligned}
& \dot{\lambda}_{1}=\lambda_{2} \lambda_{3}, \\
& \dot{\lambda}_{2}=-\lambda_{1} \lambda_{3}, \\
& \dot{\lambda}_{3}=-\lambda_{2}
\end{aligned}
$$

In addition the Casimir function (24) is again constant along the Hamiltonian flow.

Note that by setting $\lambda_{1}, \lambda_{2}=0$ in (46) we find that $\dot{\lambda}_{3}=0$ and $\lambda_{3}=$ const . Therefore this corresponds to the curves of constant curvature commonly used in motion planning. In addition, setting $\lambda_{1}, \lambda_{2}, \lambda_{3}=0$ yields straight line segments. Therefore (46) can be manipulated to obtain the motion primitives which comprise Dubins' curves.

As for the arbitrary speed case, the integrability of the unit speed system can be confirmed geometrically by plotting the intersection of the integrals of the system, the Hamiltonian (45) and the Casimir function (24) as shown in Figure 5. The two quadratic surfaces intersect and de- 


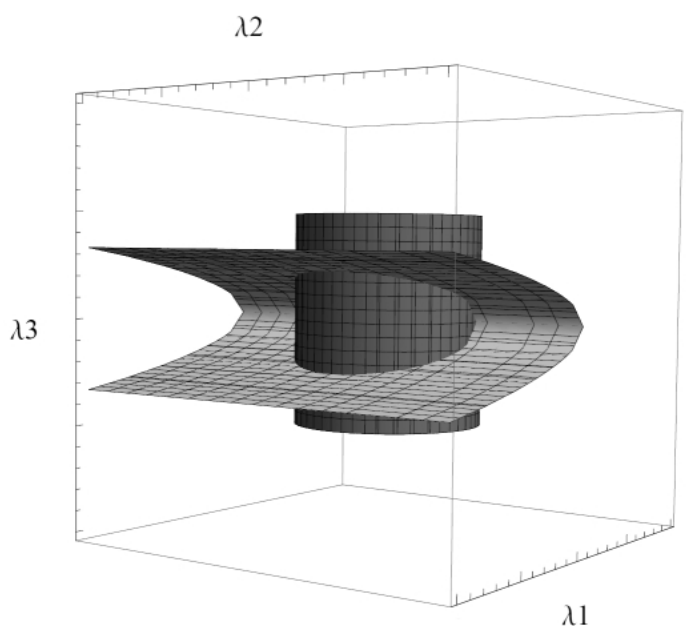

Figure 5: Plot of the Hamiltonian function (light) and Casimir (dark) in unit speed case.

fine elliptic curves which can again be parameterised by elliptic functions ${ }^{26}$. Therefore the system is integrable, and the extremal curves can be solved analytically via the use of Jacobi elliptic functions and Taylor expansions as stated in the following Lemma:

Lemma 2 The optimal angular velocity $\omega$ that minimises the cost function (41) subject to the kinematic constraint (39) is a Jacobi elliptic function of the form:

$$
\omega=\sqrt{s_{1}} \operatorname{sn}\left(\sqrt{\alpha s_{2}} t+K, \frac{s_{1}}{s_{2}}\right)
$$

where the constant $K$ is defined by:

$$
K=s n^{-1}\left(\frac{\lambda_{3}(0)}{\sqrt{s_{1}}}, \frac{s_{1}}{s_{2}}\right)
$$

with:

$$
\begin{aligned}
& s_{1}=\frac{-\beta+\sqrt{\beta^{2}-4 \alpha \chi}}{2 \alpha} \\
& s_{2}=\frac{-\beta-\sqrt{\beta^{2}-4 \alpha \chi}}{2 \alpha}
\end{aligned}
$$

and:

$$
\begin{aligned}
& \alpha=-\frac{1}{4} \\
& \beta=H^{*} \\
& \chi=M-H^{* 2}
\end{aligned}
$$

Proof. By combining the conserved quantities (45) and (24) it can be found that:

$$
\lambda_{2}=\sqrt{M-\left(H^{*}-\frac{1}{2} \lambda_{3}^{2}\right)^{2}}
$$

Then, squaring the expressions for $\dot{\lambda}_{1}$ and $\dot{\lambda}_{3}$ in (46) and combining with (51) gives:

$$
\dot{\lambda}_{3}^{2}=-\frac{1}{4} \lambda_{3}^{4}+H^{*} \lambda_{3}^{2}+\left(M-H^{* 2}\right)
$$

This expression can be written in the form:

$$
\dot{\lambda}_{3}^{2}=\alpha\left(s_{1}-\lambda_{3}^{2}\right)\left(s_{2}-\lambda_{3}^{2}\right)
$$

and then rewritten as the integral:

$$
\int_{0}^{t} d t=\int_{\lambda_{3}(0)}^{\lambda_{3}(t)} \frac{1}{\sqrt{\alpha\left(s_{1}-\lambda_{3}^{2}\right)\left(s_{2}-\lambda_{3}^{2}\right)}} d \lambda_{3}
$$

In order to simplify the integration, we use the substitution:

$$
\lambda_{3}=\sqrt{s_{1}} \operatorname{sn}(u, m)
$$

Note that $m=\frac{s_{1}}{s_{2}}$. Differentiating gives:

$$
d \lambda_{3}=\sqrt{s_{1}} \operatorname{cn}(u, m) d n(u, m)
$$

Substituting (55) and (56) into (54) leads to an equation of the form:

$$
\int_{0}^{t} d t=\int_{u_{1}}^{u_{2}} \frac{\sqrt{s_{1}} c n(u, m) d n(u, m)}{\sqrt{\alpha} \sqrt{s_{1} s_{2}-s_{1}^{2} s n^{2}(u, m)-s_{1} s_{2} s n^{2}(u, m)+s_{1}^{2} s n^{4}(u, m)}} d u
$$

where:

$$
\begin{aligned}
& u_{1}=s n^{-1}\left(\frac{\lambda_{3}(0)}{\sqrt{s_{1}}}, \frac{s_{1}}{s_{2}}\right) \\
& u_{2}=s n^{-1}\left(\frac{\lambda_{3}(t)}{\sqrt{s_{1}}}, \frac{s_{1}}{s_{2}}\right)
\end{aligned}
$$

With some manipulation equation (57) reduces to:

$$
\int_{0}^{t} d t=\int_{u_{1}}^{u_{2}} \frac{1}{\sqrt{\alpha s_{2}}} d u
$$

Integrating and rearranging leads to the equation for $\lambda_{3}(t)$ :

$$
\lambda_{3}(t)=\sqrt{s_{1}} \operatorname{sn}\left(\sqrt{\alpha s_{2}} t+K, \frac{s_{1}}{s_{2}}\right)
$$

where the constant $K$ is given in (48). Remembering that $\omega=\lambda_{3}(t)$ yields the expression for the rotational angular 
velocity (47). $\square$ The orientation of the wheeled robot is given by $\theta=\int \omega d t$, therefore:

$\theta=\frac{2 \sqrt{s_{1}}}{\sqrt{m} \sqrt{-s_{2}}} \log \left(-\sqrt{m} c n\left(K+\frac{1}{2} \sqrt{-s_{2}} t, m\right)+d n\left(K+\frac{1}{2} \sqrt{-s_{2}} t, m\right)\right)+C_{1}$ (61)

where:

$$
C_{1}=-\frac{2 \sqrt{s_{1}}}{\sqrt{m} \sqrt{-s_{2}}} \log (-\sqrt{m} c n(K, m)+\operatorname{dn}(K, m))
$$

Substituting (61) into Equation (38) yields:

$$
\frac{d \gamma}{d t}=\left[\begin{array}{c}
\cos (\theta) \\
\sin (\theta)
\end{array}\right]
$$

These expressions cannot be integrated analytically for $X$ and $Y$. However Taylor expanding (63) in $t$ about $t=0$ and integrating enables approximate analytical expressions for $X$ and $Y$ to be found. Note that as a result of the Taylor expansion these expressions will only be convergent for $t$ in $[0,1)$, however the references can be easily scaled to the desired time.

\section{Reachable Sets}

In this paper, we define reachable sets as the states in the wheeled robot's configuration space which are achievable via a single trajectory from the starting point at the origin. The robot's environment is assumed to be obstacle free when defining reachable sets.

\subsection{Arbitrary Translational and Rotational Speed}

Since the magnitudes of the forward and rotational speeds are unconstrained, the reachable sets in the $X-Y$ plane which can be reached via a single manoeuvre are limited only by the parameter space and the physical constraints of the robot (e.g. turning radius). All other $X-Y$ positions are reachable in time $t$ in the interval $(0, \infty)$. Rearranging (28) leads to an expression for the orientation $\theta_{f}$ which is reached after some final time $T$ :

$$
T=\frac{F\left(\theta_{f}, m\right)}{\alpha}
$$

where $F$ is an elliptic integral of the first kind ${ }^{30}$, with $m=M / 2 H^{*}$ and $\alpha=\sqrt{2} H^{*} / \sqrt{c H^{*}}$ as in Section 4.1. Therefore for some final orientation $\theta_{f}$ and some values of the free parameters $H^{*}, M$ and $c$, we have the time required to reach this orientation. The final position $X_{f}, Y_{f}$ can be found from (26) at $t=T$. Then for $\theta_{f} \in[0,2 \pi]$ and $H^{*}, M, c \in(0, \infty]$ the complete reachable sets for the arbitrary speed case are defined.

\subsection{Unit Speed}

The expression for $\theta(61)$ in the unit speed case cannot be solved explicitly for $t$ and so the reachable sets are difficult to analytically define. Therefore in this case it is useful to numerically evaluate time-limited reachable sets. Constraining $H^{*}, M, \lambda_{3}(0) \in(0,1]$, manoeuvre time to $T=1$ and running random Monte Carlo simulations we can numerically evaluate the time-limited reachable sets. This can be seen in Figure 6 . In this case

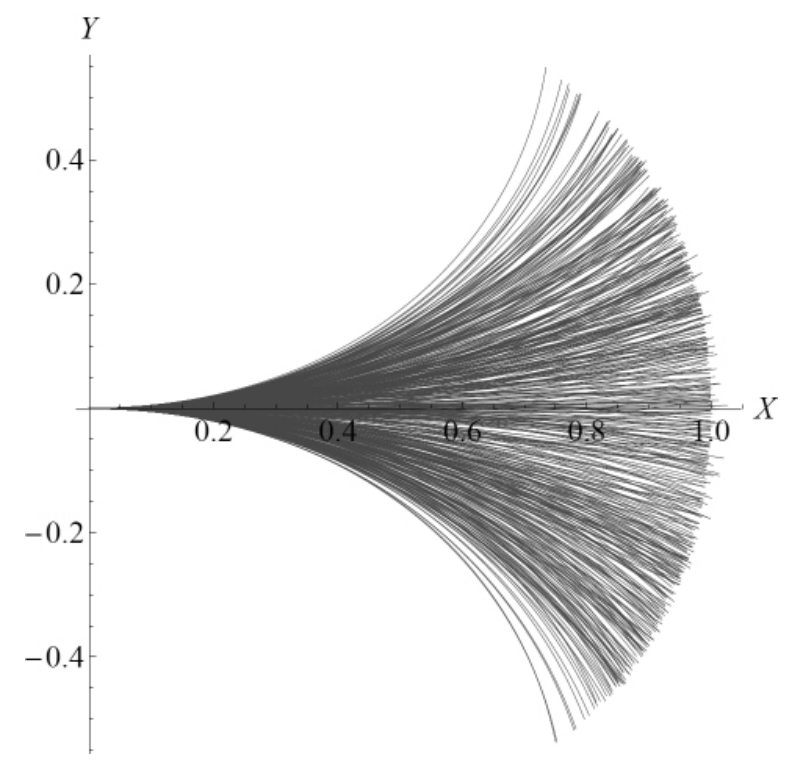

Figure 6: Time limited subset of reachable sets determined numerically for $\mathrm{T}=1, H^{*}, M, \lambda_{3}(0) \in(0,1] .1000$ trajectories plotted.

the reachable sets for the wheeled robot constrained to move at unit speed but with arbitrary rotational velocity 
are similar to the time-limited reachable sets for Dubins' car, which is constrained to move at unit speed on arcs of fixed curvature ${ }^{8}$. This is to be expected as the paths which mark the upper and lower bounds of the graph are those in which the steering angle and hence curvature are at the maximum allowable value, and so the bounds are similar to those of Dubins' car.

\section{Motion Planning and Obstacle Avoidance}

In this section we introduce a method of matching prescribed boundary conditions on the final position and orientation by parametrically optimising the free parameters in the equations derived in Section 4. We then show how the parametric optimisation can be adapted to create a simple obstacle avoidance algorithm for static obstacles in a known environment.

\subsection{Parametric Optimisation}

As the analytical expressions for the angular and translational displacements of the wheeled robot are functions of several free parameters, a parametric optimisation can be utilised in order to drive the robot to the desired target (provided the target is within the reachable sets of the planner.) However as noted by $\mathrm{LaValle}^{8}$, no natural performance metric exists on $S E(2)$ as the rotational and translational components do not have matching units. Therefore difficulties can occur when targeting both final position $(X, Y)$ and orientation $(\theta)$ using the standard Euclidean metric:

$$
\left(X-X_{d}\right)^{2}+\left(Y-Y_{d}\right)^{2}+\left(\theta-\theta_{d}\right)^{2}
$$

However, by using the complex representation of the angular displacement $\theta=a+i b$, greater accuracy can be achieved. Therefore a performance metric of the form:

$$
\min _{H^{*}, M, \Omega}\left\{\left(X-X_{d}\right)^{2}+\left(Y-Y_{d}\right)^{2}+\left(a-a_{d}\right)^{2}+\left(b-b_{d}\right)^{2}\right\}
$$

was used where $H^{*}, M$ and $\Omega$ are the free parameters to be optimised. $H^{*}$ is the optimal Hamiltonian, $M$ the Casimir function and $\Omega=c$ is a weight in the arbitrary speed case and $\Omega=\lambda_{3}(0)$ is the initial angular velocity in the unit speed case. The subscript $d$ denotes the desired or target value of the parameters $X, Y$ and $\theta$. A parametric optimisation can then be carried out to minimise the error between the current and target position in $S E(2)$ by changing the values of the free parameters. Formally this involves finding the curve $g(t) \in S E(2)$ that matches the boundary conditions $g(0) \in S E(2)$ and $g(T) \in S E(2)$ in some final time $T$.

The optimal values of the free parameters obtained as a result of the parametric optimisation are then input into the analytical expressions for the angular and translational displacements of the wheeled robot to give $\mathrm{X}-\mathrm{Y}$ position and orientation reference tracks for the robot's path. This is demonstrated in Section 6.2.

\subsection{Motion Planning and Obstacle Avoidance for Arbi- trary Translational and Rotational Speed}

In this subsection we demonstrate the planning of motions for the wheeled robot in the case of arbitrary translational and rotational speed. In the absence of obstacles, any motion which meets the prescribed boundary conditions is sufficient. Figure 7 shows position tracks for the wheeled robot, generated using the equations derived in Section 4.1, from the origin to the randomly selected points $\left[0.18,2.5, \frac{\pi}{2}\right],\left[0.4,2, \frac{\pi}{2}\right]$ and $\left[1,3, \frac{\pi}{3}\right]$ in 1 second. We see that the paths are smooth, and that the elliptic functions lead to a variety of different curves being pro- 


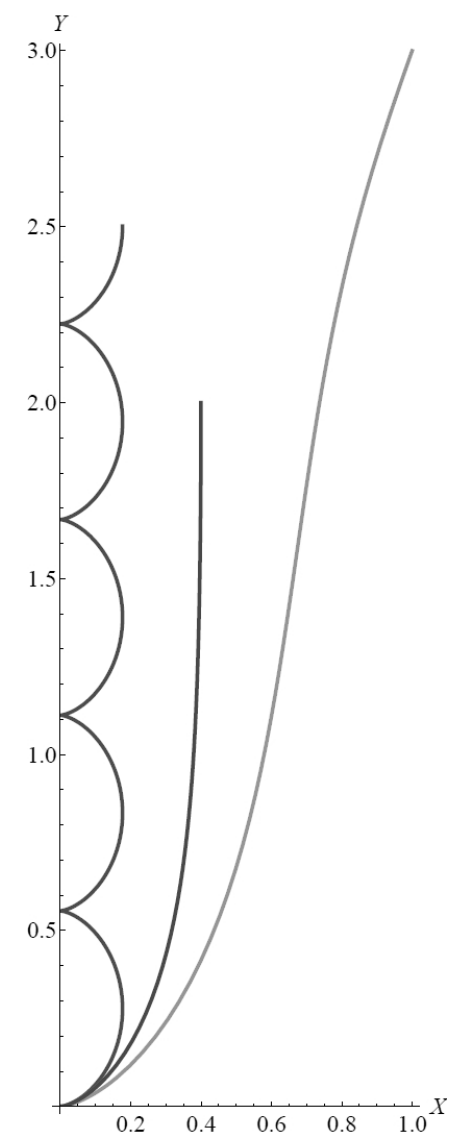

Figure 7: Smooth paths of wheeled robot in $X-Y$ space for manoeuvres to (from left to right) - $\left[0.18,2.5, \frac{\pi}{2}\right]$, $\left[0.4,2, \frac{\pi}{2}\right],\left[1,3, \frac{\pi}{3}\right]$

duced as the free parameters are varied.

In reality, the configuration space may contain obstacles. In this case the set of all curves which match the boundary conditions on $X$ and $Y$ can be probed to determine if a suitable curve exists which avoids the obstacle. Figure 8 shows a subset of the curves which match a target position of $[1,3]$ in a time of 1 second. It is obvious that there are multiple curves which satisfy the position constraint. In the simplest case, where the obstacle is considered to be a point mass, the curves must not violate the condition:

$$
\sqrt{\left(X-X_{o}\right)^{2}+\left(Y-Y_{o}\right)^{2}}=\chi
$$

where the subscript $o$ refers to the position of the obstacle and $\chi \neq 0$ for obstacle avoidance. In reality, a for-

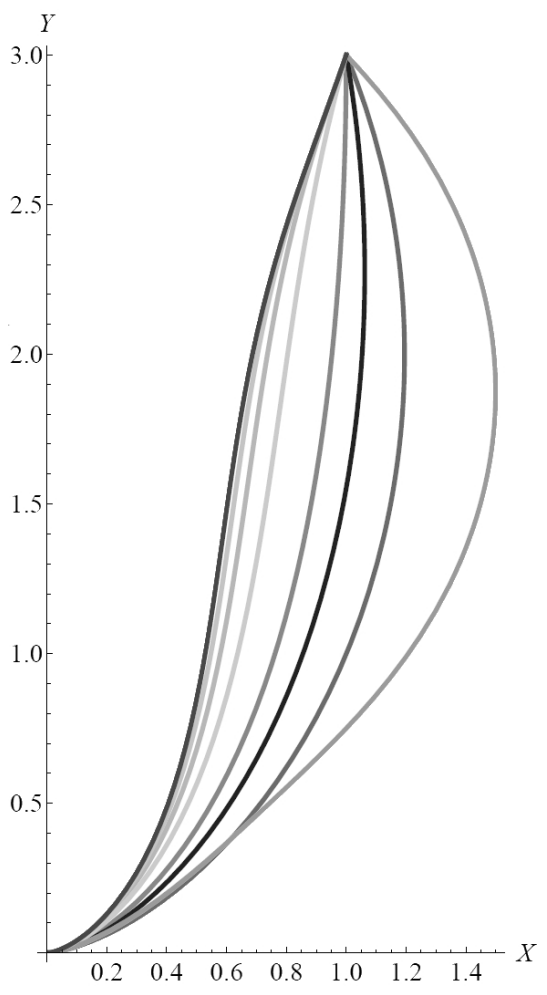

Figure 8: Illustration of a subset of curves which match $[1,3]$ boundary conditions

bidden "zone" is created in the configuration space, and so a range of positions must be avoided during the manoeuvre. Considering the obstacle to be stationary and represented by a circle of radius $\chi_{o}$ in the $X-Y$ configuration space, the condition for obstacle avoidance is then given by (67) where $\chi>\chi_{o}$. Given the proposed method outlined in Section 6.1 for the analytical expressions defined in Section 4.1, the parametric optimisation will return the optimal values of the free parameters $H^{*}, M^{*}, c^{*}$ which satisfy the target position. In the case that this manoeuvre violates $\chi>\chi_{o} \forall t$, a simple algorithm can be devised to overcome this. Defining the forbidden region as a circle in $S E(2)$ with radius $\chi_{o}$ and centre $\left[X_{o} Y_{o}\right]^{T}$, provided $\chi>\chi_{o} \forall t$ the trajectory will avoid the forbidden region. If $\chi<\chi_{o}$ for $H^{*}, M^{*}, c^{*}$ for any $t$ in the interval $(0, T)$ then we further optimise parameters $p_{1}, p_{2}, p_{3}$ such that $p_{1}^{2}>\eta, p_{2}^{2}>\eta, p_{3}^{2}>\eta$, where $\eta>0$ is a small 
parameter. This leads to a new set of free parameters $H^{* *}=H^{*}+p_{1}, M^{* *}=M^{*}+p_{2}, c^{* *}=c^{*}+p_{3}$ being obtained, with the objective function:

$$
\min _{p_{1}, p_{2}, p_{3}}\left\{\left(X-X_{o}\right)^{2}+\left(Y-Y_{o}\right)^{2}\right\}
$$

such that $\chi>\chi_{o} \forall t$. Note that $\eta$ can be tuned until $\chi>\chi_{o} \forall t$ is satisfied. We follow with an example.

For a manoeuvre from the origin to $[1,3]$ with a static obstacle centred at $\left[X_{o}, Y_{o}\right]=[0.66,1.52]$ and with radius $\chi_{f}=0.3$, the following approach could be employed. First, generate a manoeuvre using the motion planning method described in Section 6.1 above. Then, check to see if the condition $\chi>\chi_{o} \forall t$ is violated at any time step. For instance, for the 1 second repointing manoeuvre to $\left[1,3, \frac{\Pi}{3}\right]$ shown in Figure 7 we find that the wheeled robot collides with the obstacle at $t=0.56$ seconds. Figure 9 illustrates this. Now, a new trajectory can be generated by

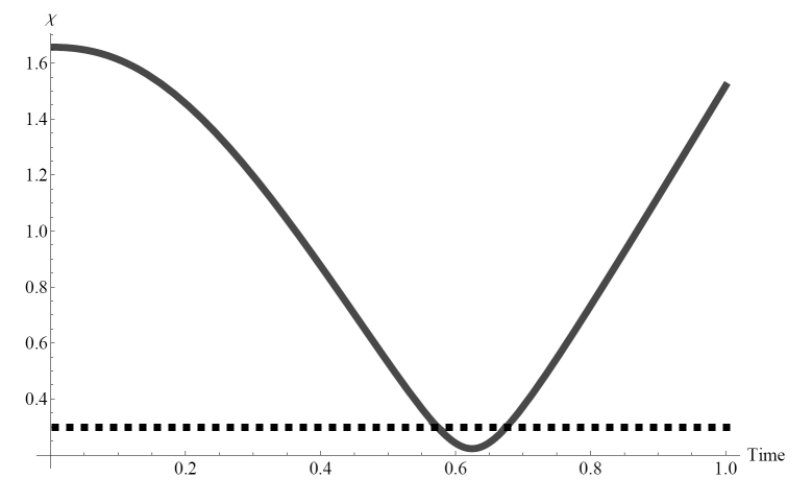

Figure 9: Error between current position and centre of forbidden zone of radius $\chi_{o}$ (solid line). Horizontal (dashed) line marks radius of obstacle. Constraint is violated.

using the parameters $p_{i}$ (where $\left.i=1, \ldots, 3\right)$ to produce a new set of free parameters which drive the wheeled robot to the target via (68). This process is repeated, tuning the value of the parameter $\eta$ until an appropriate solution is found which does not violate the constraint and still meets the desired final position to high precision. One such solution for a constrained manoeuvre from the origin to $[1,3]$ is shown in Figures 10 and 11.

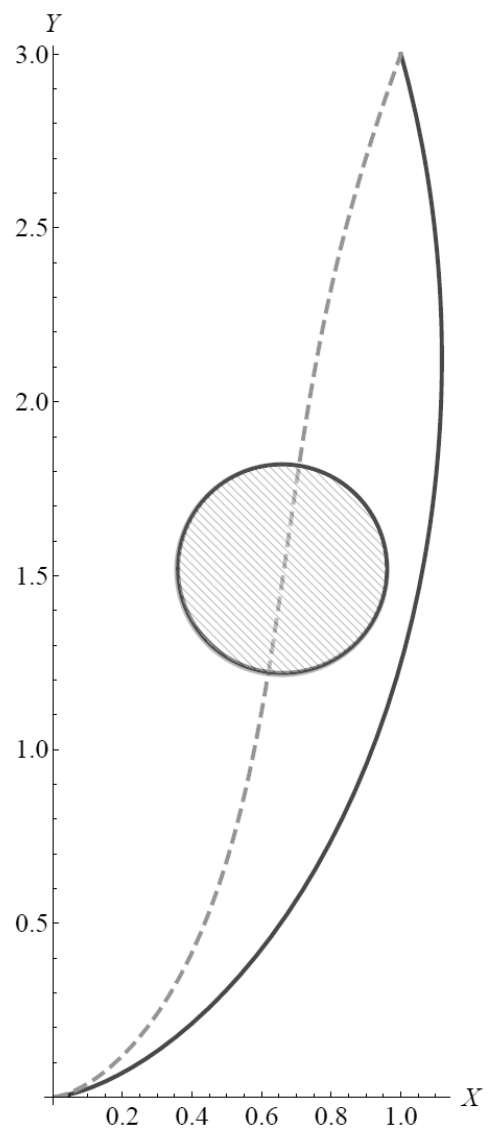

Figure 10: Alternative smooth trajectory for manoeuvre to $[1,3]$ (solid line). Also shown is the original path (dashed line) intersecting with the obstacle (shaded circle).

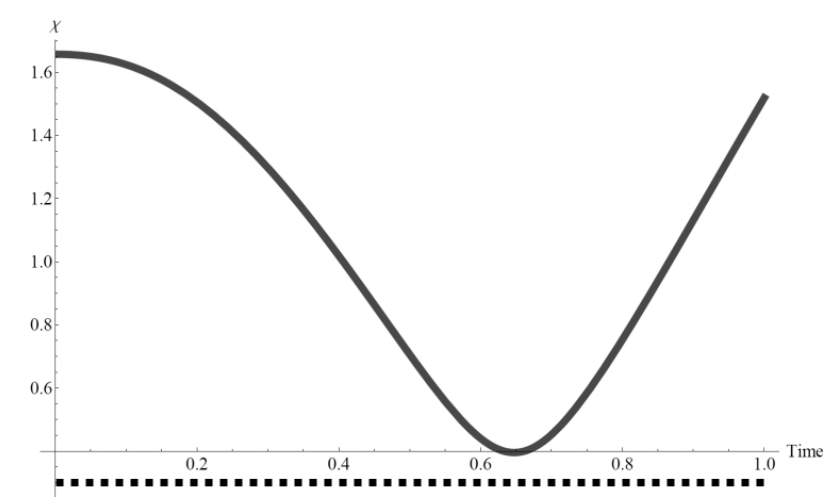

Figure 11: Error between current position and centre of forbidden zone of radius $\chi_{o}$ (solid line). Horizontal (dashed) line marks radius of obstacle. Constraint is not violated since $\chi>\chi_{o} \forall t$. 
It is evident that the constraint $\chi>\chi_{o} \forall t$ is respected and therefore the wheeled robot would not collide with the specified obstacle.

Note that while the obstacle avoidance algorithm has been demonstrated here using the arbitrary speed case, the analytical expressions for position yielded via Taylor expansion in the unit speed case enable the obstacle avoidance algorithm to be applied in this case also. However the forward speed constraint in this case limits the number of alternative trajectories to the same position. Also note that while the obstacle avoidance algorithm was implemented for static obstacles in a known environment, the analytical curves can be generated swiftly and efficiently and so an extension to dynamic obstacles in uncertain environments may be feasible in future.

The main limitation of the obstacle avoidance method detailed above is that while alternative trajectories can be generated to a specified position in the $X-Y$ plane, it is not always possible to generate an alternative curve which does not collide with the obstacle and which satisfies both a position $(X, Y)$ and an orientation $(\theta)$ constraint. For example in Figure 10 the position constraint $[1,3]$ is respected, but the final orientation differs from that of the original curve.

In the case where an alternative curve has been generated in the presence of an obstacle and a desired final orientation is not achievable via a single curve, or indeed if a desired final state cannot be reached due to sliding and turning constraints, a more complex motion planning algorithm is required. For example while a single curve generated via the motion planner described in this paper may not be sufficient in all cases, a combination of curves generated by the motion planner and straight line segments may enable the desired final state to be reached. The mo- tion planning methodology can then be summarised in the following stages:

1. Use the motion planning method described in Section 6.1 to minimise the error between the actual and desired final state $\left(X_{d}, Y_{d}, \theta_{d}\right)$ by changing the values of the free parameters in the analytical expressions derived in Sections 4.1 and 4.2. If the accuracy of the solution is sufficiently high, and it does not intersect with any obstacles in the configuration space, the trajectory is suitable.

2. In the case where the trajectory intersects with a static, known obstacle, and where final orientation is not of paramount importance, further optimise the parameters $p_{i}$ as described above to generate an alternative trajectory which does not collide with the obstacle and which sufficiently satisfies the desired final position constraint $\left(X_{d}, Y_{d}\right)$.

3. If no single suitable trajectory is found use a combination of curves generated by motion planner and straight lines to achieve the desired final state.

The difficulty in piecing together motions lies in ensuring that the transition between segments is smooth (as in the work of Scheuer and Fraichard ${ }^{13}$ ) and that there are no infeasible increases in translational and rotational speeds between sections. Several methods for achieving this have been proposed ${ }^{31-34}$. These methods consider the workspace of the robot and any obstacles therein, and then query the reachable sets to create feasible paths for the robot by concatenation of different motion primitives. However this process is outwith the scope of this paper. 


\section{Conclusions}

In this paper a motion planning method for simple wheeled robots with a sliding constraint was derived using optimal control theory for systems defined on Lie groups. Two cases were considered: (i) the case where translational and rotational speeds are arbitrary and; (ii) the case where the robot is constrained to move forwards at unit speed.

It was found that in the arbitrary speed case the optimal controls take the form of elliptic functions. This class of function degenerates to simple trigonometric functions under certain conditions, and so a wide range of curve types are available for motion planning.

In the unit speed case the rotational velocity was described in terms of elliptic integrals and the expression for the position reduced to quadratures. A truncated approximation was then used to describe the position analytically, resulting in paths which trace elastic curves.

Reachable sets were defined analytically in the arbitrary speed case by expressing the final manoeuvre time as an elliptic integral of the first kind, and were illustrated numerically in the unit speed case where the time-limited reachable sets resembled those of Dubins' car over short time spans. It was shown that the analytical expressions for position and orientation can be exploited to efficiently generate smooth and feasible paths for the wheeled robots via parametric optimisation. Finally an algorithm for introducing obstacle avoidance into the parametric optimisation procedure was presented for static obstacles in a known environment. In this procedure a further parametric optimisation probes the reachable sets to find a curve which avoids the static obstacle while satisfying a position constraint.

Future work will see the implementation of a concatena- tion method to feasibly piece together the curves of the motion planner to give a more complete motion planning algorithm. This will include amending the motion planning method to have non-zero initial speeds, and developing an algorithm to choose the paths most suited to being pieced together. In addition the possibility of extending the simple obstacle avoidance algorithm described above to dynamic and unknown environments will be explored.

\section{References}

[1] V. Jurdjevic, Geometric Control Theory (Advanced Studies in Mathematics, Cambridge University Press, 52, 1997).

[2] A. M. Bloch, Nonholonomic Mechanics and Control (SpringerVerlag, 2003).

[3] N. Leonard and P. S. Krishnaprasad, 'Motion control of drift free, left-invariant systems on Lie groups,' IEEE Trans. Autom. Control, 40, 1539-1554, (1995).

[4] J. Biggs and N. Horri, 'Optimal geometric motion planning for spin-stabilized spacecraft,' Systems and Control Letters, 61(4), 609-616, (2012).

[5] J. Biggs, 'Optimal path planning for nonholonomic robotics systems via parametric optimisation,' Proceedings of TAROS 2011, Sheffield, (2011).

[6] V. G. Boltyanskii, R. V. Gamkrelidze and L.S. Pontryagin, 'Towards a theory of optimal processes,' Reports Acad. Sci. USSR, 110(1), 7-10, (1956).

[7] L. E. Dubins, 'On curves of minimal length with a constraint on average curvature, and with prescribed initial and terminal positions and tangents,' American Journal of Mathematics, 79, $497-$ 516 , (1957).

[8] S. M. LaValle, Planning Algorithms (Cambridge University Press, 2006).

[9] V. Jurdjevic and F. Monroy-Perez, 'Variational problems on Lie Groups and their homogeneous space: Elastic curves, tops, and constrained geodesic problems,' In: Contemporary Trends in Nonlinear Geometric Control Theory and its Applications A. AnzaldoMeneses, B. Bonnard, J. P. Gauthier and F. Monroy-Perez Eds., (World Scientific, 2002).

[10] J. A. Reeds and L. A. Shepp, 'Optimal paths for a car that goes both forwards and backwards,' Pac. J. Math, 145(2), 367-393, (1990). 
[11] A. Tsourdos, B. A. White and M. Shanmugavel Cooperative Path Planning of Unmanned Aerial Vehicles (Wiley, 2011).

[12] N. Mahmoudian and C. Woolsey, 'Underwater glider motion control,' Proceedings of the 47th IEEE Conference on Decision and Control, Cancun, Mexico (2008) pp. 552-557.

[13] A. Scheuer and Th. Fraichard, 'Continuous curvature path planning for car-like vehicles,' Proceedings of the IEEE International Conference on Intelligent Robots and Systems, Montbonnot-SaintMartin, France (1997) vol. 2, pp. 997-1003.

[14] A. Scheuer and Th. Fraichard, 'Collision-free and continuouscurvature path planning for car-like robots,' Proceedings of International Conference on Robotics and Automation, Albuquerque, New Mexico (1997) pp. 867-873.

[15] Th. Fraichard and J. M. Ahuactzin 'Smooth path planning for cars,' Proceedings of International Conference on Robotics and Automation, Seoul, South Korea (2001) vol. 4, pp. 3722-3727.

[16] R. Murray and S. Sastry, 'Steering nonholonomic systems using sinusoids,' Proceedings of 29th IEEE Conference on Decision and Control, Honolulu, Hawaii (1990) pp. 2097-2101.

[17] R. W. Brockett, 'Control theory and singular Riemannian geometry,' In: New Directions in Applied Mathematics, pp. 11-27, (Springer-Verlag, 1981).

[18] A. Kelly and B. Nagy, 'Reactive Nonholonomic Trajectory Generation via Parametric Optimal Control,' Int. J. Rob. Res, 22(7-8), 583-601, (2003).

[19] R. Murray, Z. Li and S. Sastry, A Mathematical Introduction to Robotic Manipulation (CRC Press, 1994).

[20] J. F. Canny, The Complexity of Robot Motion Planning (Cambridge, MA: MIT press, 1988).

[21] J. C. Latombe, Robot Motion Planning (Boston: Kluwer, 1991).

[22] R. W. Brockett and L. Dai, 'Non-holonomic kinematics and the role of elliptic functions in constructive controllability,' In: Nonholonomic Motion Planning, (Kluwer Academic Publishers, 1993).

[23] H. Choset, K. Lynch, S. Hutchinson, G. Kantor, W. Burgard, L. Kavraki and S. Thrun, Principles of Robot Motion - Theory, Algorithms, and Implementation (Boston, MA: MIT press, 2005).

[24] H. J. Sussmann, 'An introduction to the coordinate-free maximum principle,' In: Geometry of Feedback and Optimal Control B. Jakubczyk and W. Respondek Eds., 463-557 (Marcel Dekker, New York, 1997).

[25] V. Jurdjevic, 'Hamiltonian point of view of non-Euclidean geometry and elliptic functions,' Systems and Control Letters, 43, 25-41,
(2001).

[26] D. Husemoller, Elliptic Curves (Springer, 2004).

[27] F. W. J. Olver, D. W. Lozier, R. F. Boisvert and C. W. Clark, NIST Handbook of Mathematical Functions (Cambridge University Press, 2010).

[28] K. R. Meyer, 'Jacobi elliptic functions from a dynamical systems point of view,' The American Mathematical Monthly, 108(8), 729737, (2001).

[29] V. Jurdjevic, 'Non-Euclidean elastica,' American Journal of Mathematics, 117(1), 93-124, (1995).

[30] M. Abramowitz and I. A. Stegun, Handbook of Mathematical Functions with Formulas, Graphs, and Mathematical Tables (Dover, 1972).

[31] E. Mazer, J. M. Ahuactzin, E. G. Talbi and P. Bessiere, 'Robot motion planning with the Ariadne's Clew algorithm,' Proceedings of the International Conference on Intelligent Autonomous Systems, Pittsburgh (1993).

[32] P. Svetska and M. H. Overmars, 'Probabilistic path planning,' Technical Report UU-CS-1995-22, Utrecht University, Utrecht, The Netherlands (1995).

[33] E. Frazzoli, M. A. Dahleh and E. Feron, 'Maneuver-based motion planning for nonlinear systems with symmetries,' IEEE Trans. Robot., 21(6), 1077-1091, (December 2005).

[34] M. Pivtoraiko and A. Kelly, 'Generating near minimal spanning control sets for constrained motion planning in discrete state spaces,' Proceedings of the IEEE/RSJ International Conference on Intelligent Robots and Systems, IROS(2005), pp. 3231 - 3237. 